\title{
Direct Iterative Reconstruction of Computed Tomography Trajectories (DIRECTT)
}

\author{
Axel Lange, Manfred. P. Hentschel \\ Federal Institute for Materials Research and Testing (BAM), Berlin, Germany \\ BAM-VIII.32; Phone: 0049308104 3667, 00493081041832 \\ e-mail:axel.lange@bam.de manfred.hentsch@bam.de
}

\begin{abstract}
The direct reconstruction approach employs an iterative procedure by selection of and angular averaging over projected trajectory data of volume elements. This avoids the blur effects of the classical Fourier method due to the sampling theorem. But longer computing time is required. The reconstructed tomographic images reveal at least the spatial resolution of the radiation detector. Any set of projection angles may be selected for the measurements. Limited rotation of the object yields still good reconstruction of details. Projections of a partial region of the object can be reconstructed without additional artifacts thus reducing the overall radiation dose. Noisy signal data from low dose irradiation have low impact on spatial resolution. The image quality is monitored during all iteration steps and is pre-selected according to the specific requirements. DIRECTT can be applied independently from the measurement equipment in addition to conventional reconstruction or as a refinement filter.
\end{abstract}

Keywords: computed tomography, reconstruction algorithm

\section{Introduction}

In medical diagnostics and material science computerized tomography provides section images of the inner structure of objects by reconstruction of radiographic projections. In the classical approach the Fourier filtered back projections of the transmission data are computed [1]. This requests the irradiation of an object at numerous angles of a full rotation or at least of $180^{\circ}$ in case of parallel beam projections. The Fourier Method, including several refinements for 3-D reconstruction of cone beam measurements (Feldkamp algorithm [2]), represents the state of the art since several decades. In case of large objects full rotation is often prohibited due to the given sample size and geometry especially if the desired enlargement requires small sample to source distance. The tomographic images are less precise than the spatial resolution of the detector due to the necessary averaging over at least two detector pixel positions (Nyquist's theorem [3]). The correct imaging of regions of interest requires the irradiation (and reconstruction) of the whole object. Low radiation doses create considerable blur.

In order to overcome these disadvantages a new algorithm without Fourier filtering has been created. It is based on the iterative analysis of the projection data (sinograms) [4]. The new iterative algorithm performs the tomographic reconstruction of projections at the resolution of detector element spacing or better.

It should be noted that it is different from algebraic reconstruction techniques (ART) although some analogies occur. DIRECTT does not employ solutions of linear equation systems.

\section{Reconstruction principle}


Using parallel beam projections for simplicity the most predominant sinusoidal trajectory projections of sample elements are selected referring either to their contrast or their intensity (Fig.1). Each sinus path corresponds to exactly one element of the reconstruction matrix (image pixel). Fractions of their weight or contrast are added at the calculated image position (reconstruction matrix). The preliminary reconstruction matrix is then projected into a sinogram, which is subtracted from the original one. The resulting residual sinogram is then iteratively treated like the original data set.

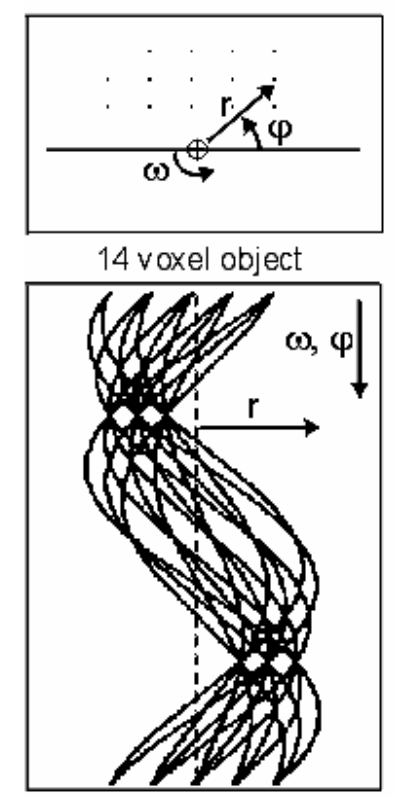

parallel beam sinogram

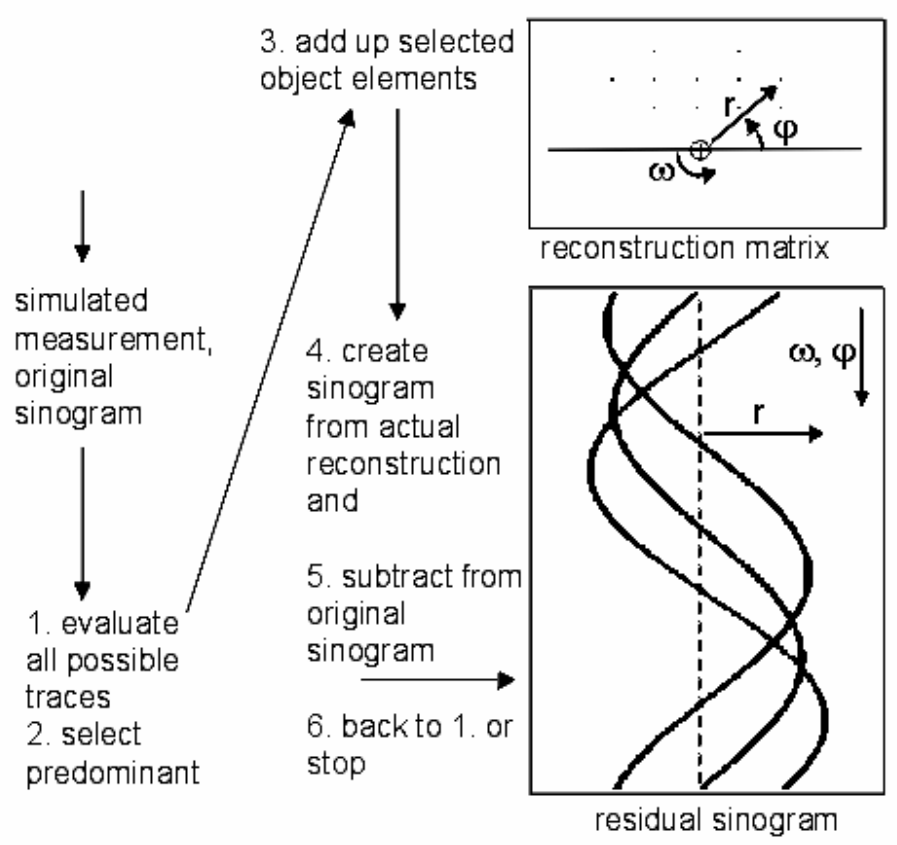

Figure 1. Reconstruction principles of the iterative procedure

As soon as the weight of the residual sinogram has become weak enough after sufficient iterations nearly all the sample information is collected into the reconstruction. The integral of the remaining sinogram contains exactly the information which was not used for the reconstruction and is therefore a quality indicator. The predominant advantage of the new algorithm is a sharp image at detector pixel size resolution, but longer computing time is still needed [4].

\section{Modeling, complete data sets}

For testing purposes a C-program on a standard PC serves to reconstruct a model structure, the "box phantom" containing different density levels, homogeneous areas and the DIRECTT acronym with dotted letters (Fig.2, left). From this a sinogram of parallel projections is created to serve as a simulated data set. The conventional reconstruction by Fourier back projection is created for reference (Fig.2, middle). The reconstruction by 200 iterations of DIRECTT and the original model are visually identical (Fig.2, right). The DIRECTT reconstruction reveals exactly the resolution of the (simulated) detector pixel size (distance). The linear resolution of the conventional reconstruction is two times worse. 
The quality of the DIRECTT reconstruction raises during the iterative procedure of the algorithm as demonstrated by different numbers of iteration steps (Fig. 3). After 125 iterations (bottom, left) the visual quality of the reconstruction exceeds already the conventional performance. The first 50 iteration apply solely weights, the followed by contrast iterations.

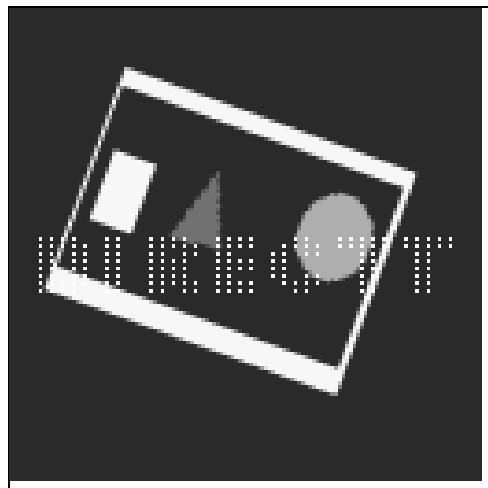

model (128 pixel) $)^{2}$

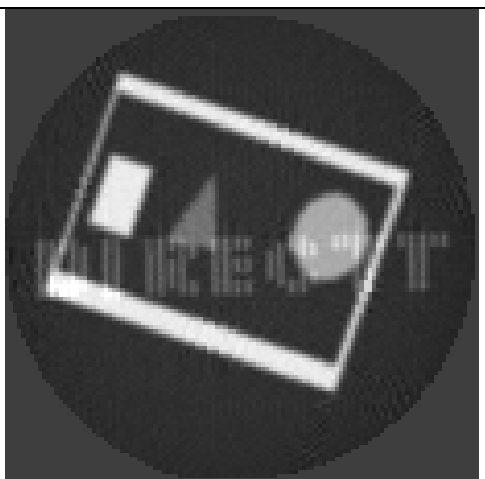

Fourier back projection computing time $\simeq 3 \mathrm{sec}$

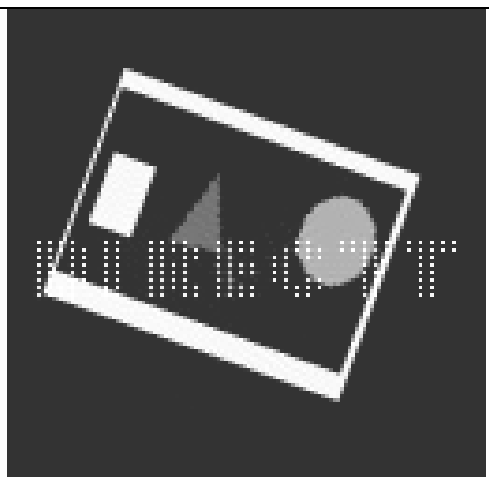

DIRECTT, 200 iterations computing time $\simeq 1 \mathrm{~min}$

Figure2. "box" phantom, Fourier filtered back projection and DIRECTT reconstruction.

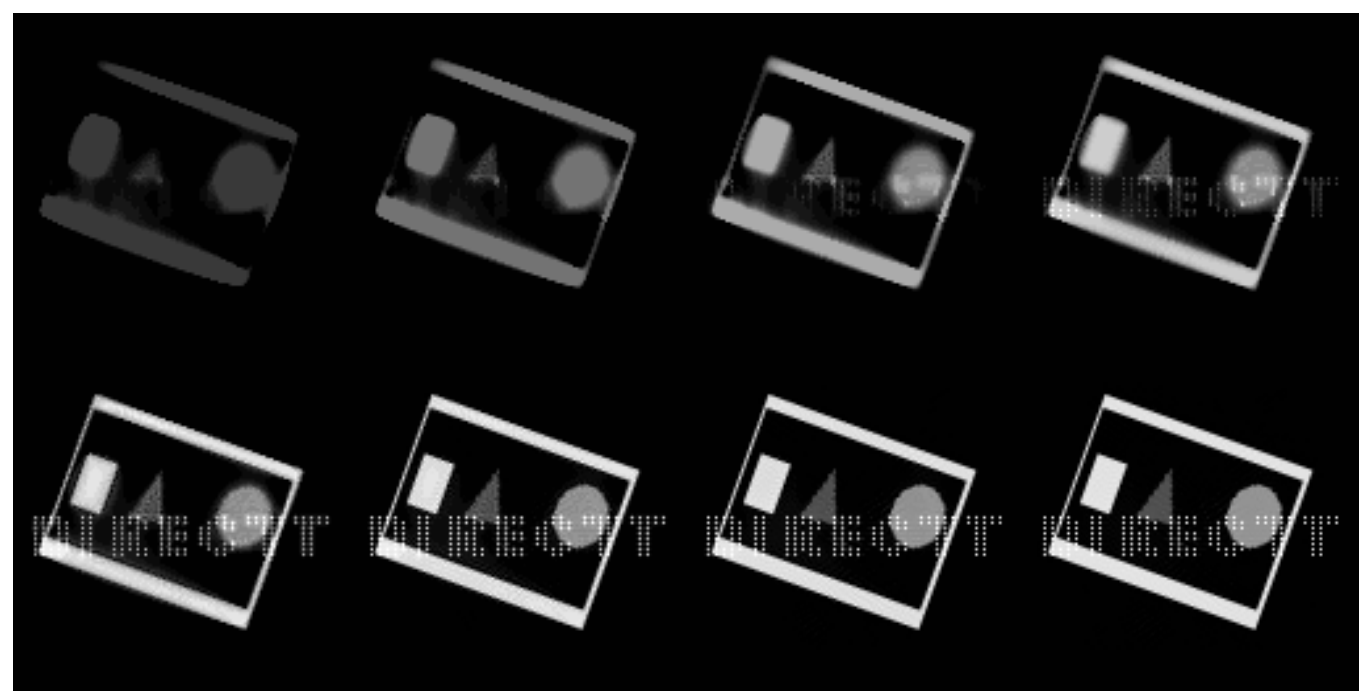

Figure 3. Evolution of DIRECTT reconstructions by steps of additional 25 iterations.

The information on the quality of the reconstruction is contained in the residual sinogram which appears after each iteration step. The simplest parameters to be extracted for this purpose are the average (weight) and the variance, which contains the information of the fine structure.

They can be recorded during the iteration process (Fig. 4). Thus the reconstruction quality can be monitored and may be pre-selected. If all weights are reconstructed 
within one iteration cycle, the resulting image is heavily blurred like unfiltered back projections (Fig. 5, left). The complete reconstruction of contrast by one iteration cycle reveals highly contrasted edges but no mass (Fig. 5, right).

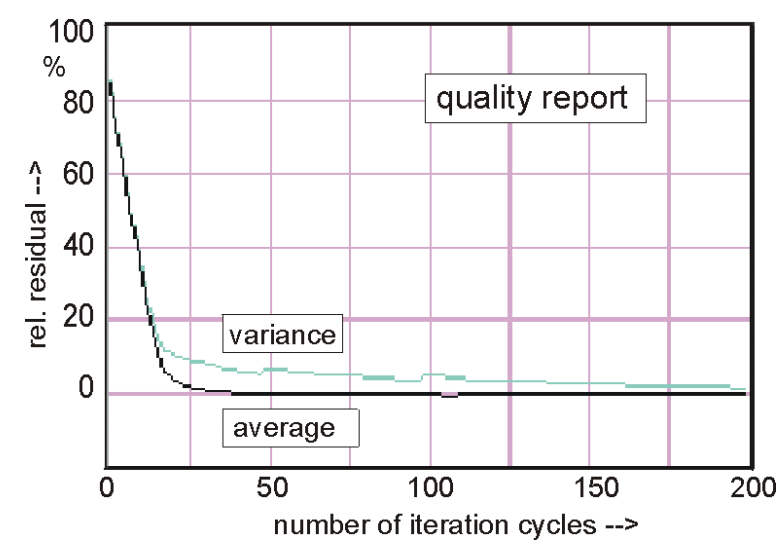

Figure 4. Report on the reconstruction quality (of example in Fig. 3): average and variance of the residual sinograms over the number of iteration cycles.
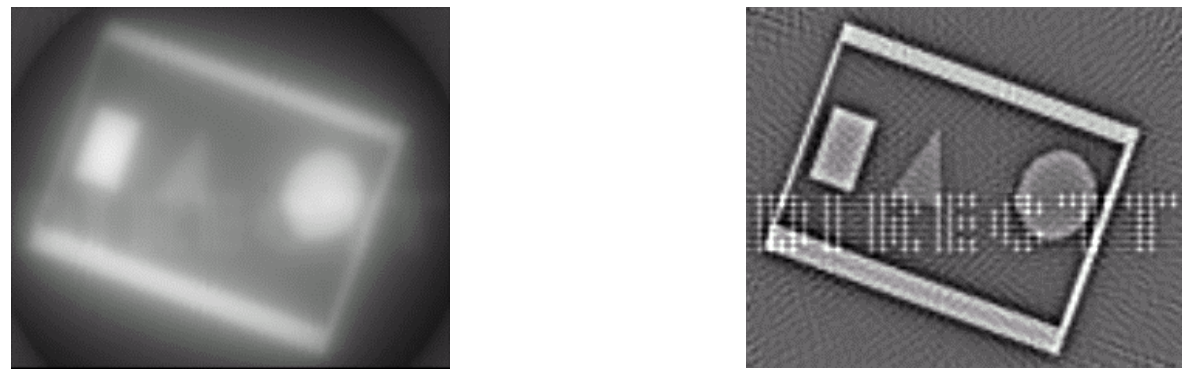

Figure 5. DIRECTT reconstructions of box model after 1 iteration cycle; left : all weights; right all contrasts

\section{Modeling of incomplete data sets}

A further advantage of DIRECTT is its capability to reconstruct projections of an arbitrary selection of rotation angles without the well known stripe artifacts of a conventional reconstructions. The first example models the reconstruction from a reduced number of projections over 180 degree, e.g. 15 projections at $12^{\circ}$ intervals, $30 \mathrm{x}$ $6^{\circ}$ and $60 \times 3^{\circ}$.

Fig. 6 shows the reconstructions of both conventional (top) and DIRECTT (bottom). The typical stripe artifacts are absent in the new method and details are much sharper.

Another case of an incomplete set of projections refers to a limited sector of projection angles. This is relevant to applications, where full sample rotation is not possible due to geometrical limitations.

Fig. 7 shows such a series of reconstructions comparing conventional (top row) and DirecTT (bottom). The angular sector varies from $60^{\circ}$ to $170^{\circ}$ (at $1^{\circ}$ steps). The 
DIRECTT algorithm reduces artifacts and details are well reconstructed from a $90^{\circ}$ angular range. The densities inside homogeneous areas correspond better to the original.

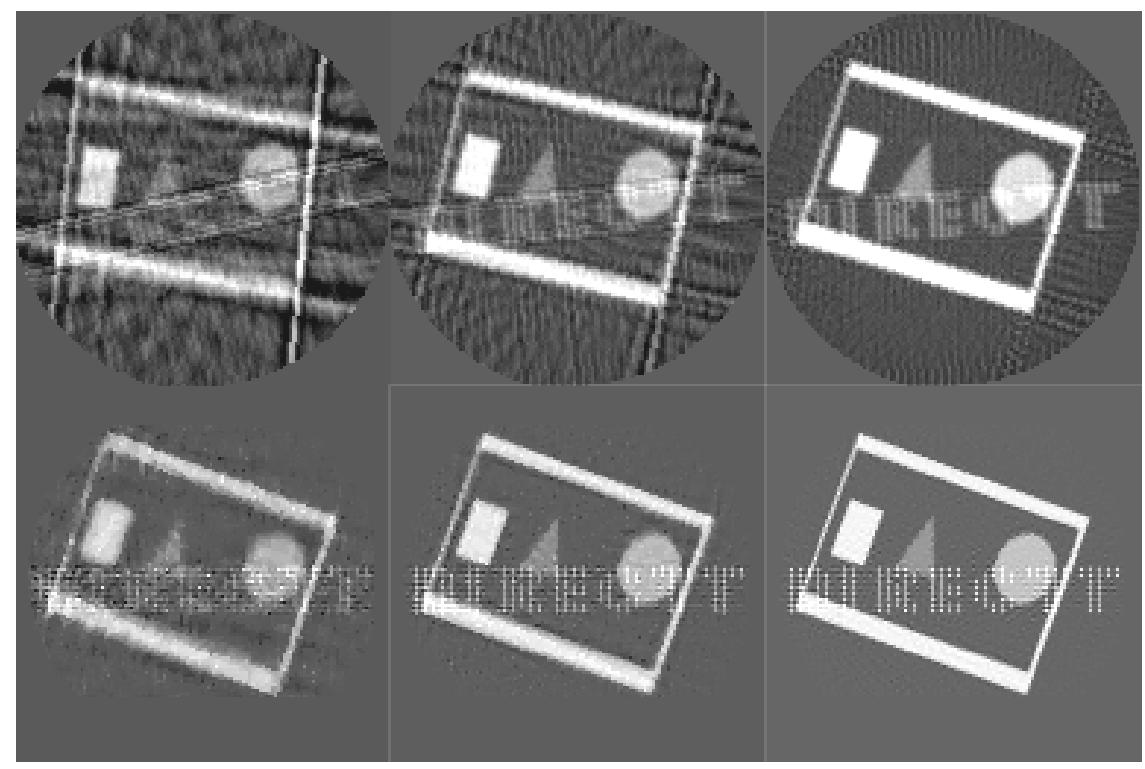

Figure 6. Reconstruction results of both conventional (top) and DIRECTT (bottom) by reduced number of projections; the rotation intervals are constant over 180 degree, e.g. 15 projections of $12^{\circ}$ separation, $30 \times 6^{\circ}$ and $60 \times 3^{\circ}$ (left to right).

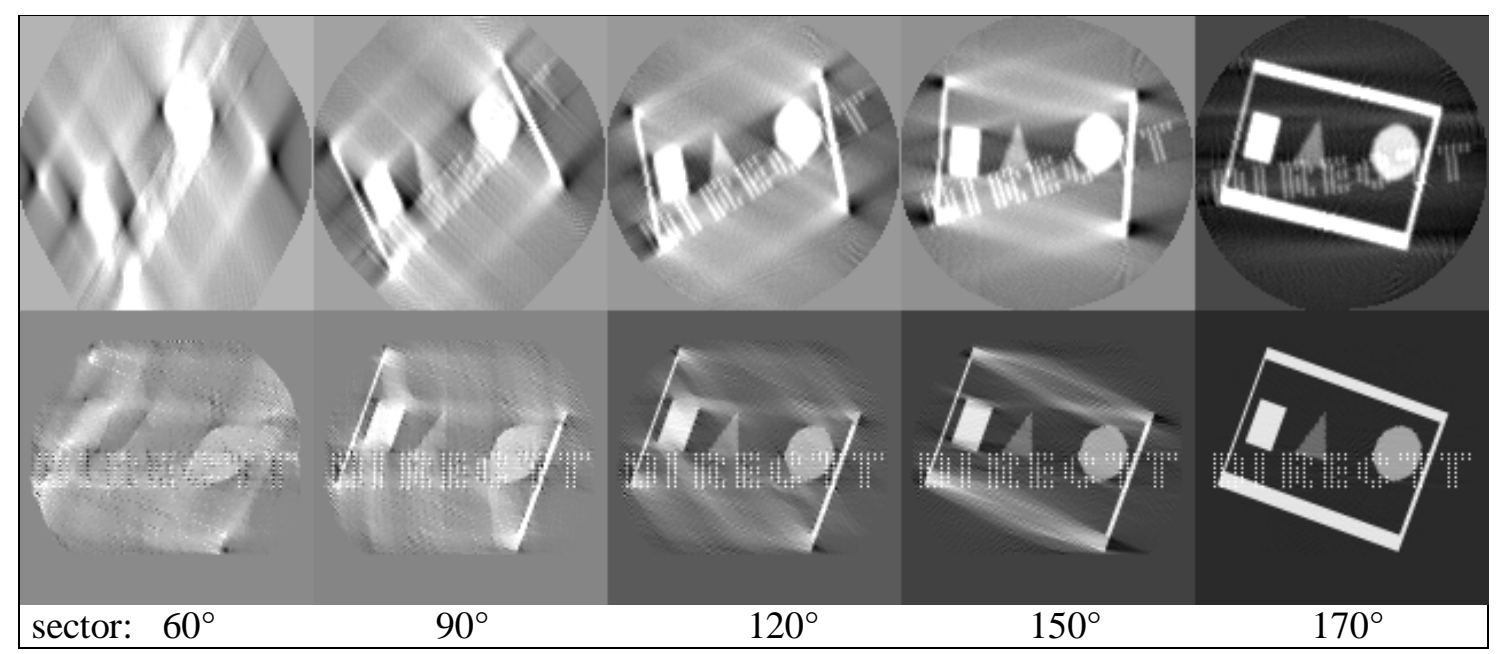

Figure 7. Reconstructions from projections of limited sectors comparing conventional (top row) and DIRECTT (bottom).

Another essential issue relates to the possibilities to expose selectively only a region of interest (ROI) of a sample and reconstruct this without the well known disadvantages of the conventional Fourier method. This is interesting for the purpose of magnification inside undesired large objects or as well as to avoid an unnecessary irradiation in medical applications. Fig. 8 demonstrates the capabilities of DIRECTT to reconstruct precisely the density and structural details of a region of interest without exposing the hole sample. 


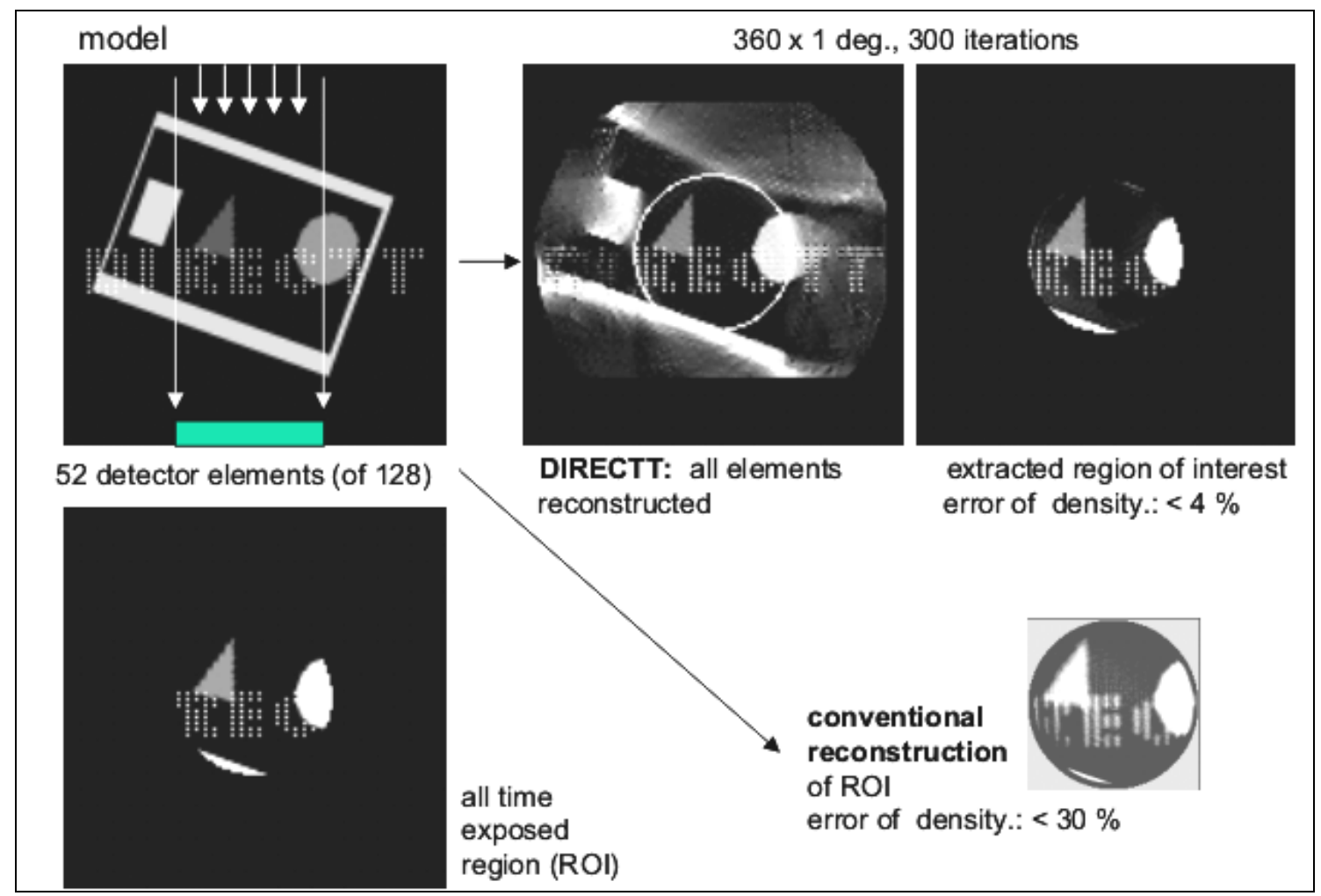

Figure 8. Reconstruction from projections of a partial exposure (region of interest, ROI); model (left), ROI reconstructed by DIRECTT (top middle and right) and conventional (bottom, middle).

Generally noise has no direct influence on the spatial resolution as the reconstruction procedure averages the sinogram data directly over the projection angles, not over the projection coordinate. Sharp contrasts are retained, although density noise occurs.

\section{Sub detector resolution}

Due to its precise reconstruction principles DIRECTT provides higher resolution than the detector, if this is the predominant component for the resolution of the projection
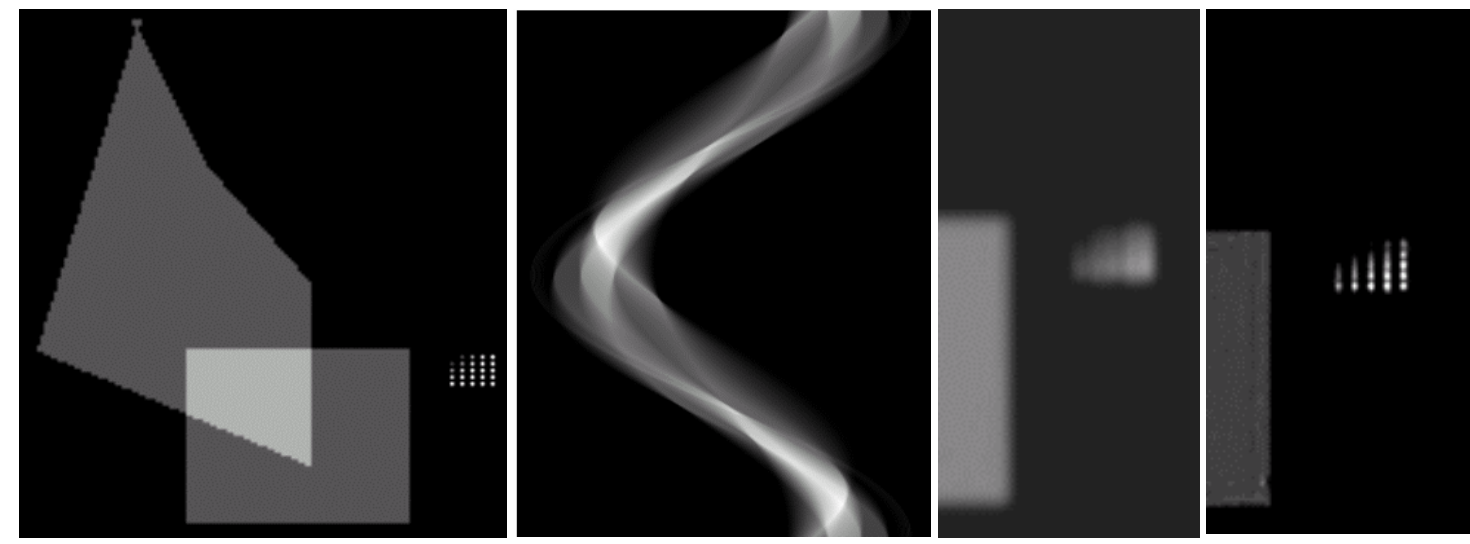

Figure 9. Sub-pixel size resolution; model with $2 \times 3$ pixel grid, sinogram after 2 pixel binning, magnified section of Feldkamp reconstruction, DIRECTT reconstruction (from left to right) 
(like in parallel beam or low magnication projection). This is demonstrated by a reconstruction program for fan beam projections applied to a model of homogeneous areas and structures with a density gradient (Fig. 9, left). After a two pixel binning of the original sinogram the reconstructions are performed by the Feldkamp and DIRECTT method (Fig. 9, right). The fine structure reconstruction of the pixel array by DIRECTT reveals a four times better resolution than Feldkamp.

This is extended towards a 4 fold detector binning for a simpler model structure of 5 elements (Fig.10 up left ). While the Fourier filtered backprojection results in a cloudy reconstruction at the right position the DIRECTT method clearly can resolve the dots. Aditionally a focal smear of the indicated size was applied. The remaining sinograms of each state, original , 4 fold binning, and additional focal smear are indicated down right(Fig.10). While minor changes of the resultant reconstruction can be seen, this time the concurrent method was omitted because of being even more smeared. So on total the resulting spatial resolution is up to 64 times better than the Fourier filtered backprojection. In case of measuring the focal smear it seems possible to apply this in the reconstruction and to get better results. Of course, the detector is assumed to be ideal. It seems clearly to be worth the price of calculating 80 Iterations to get this result.

simulation by 4-fold binning of model sinograms

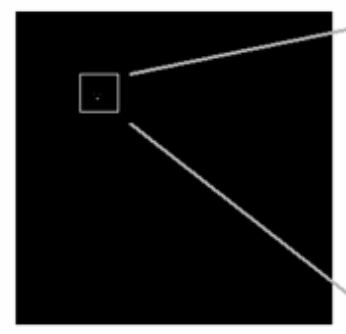

5 dot model, $256^{2}$ pixel, $720 \times 1 / 4^{\circ}$

binning and focal smearing:

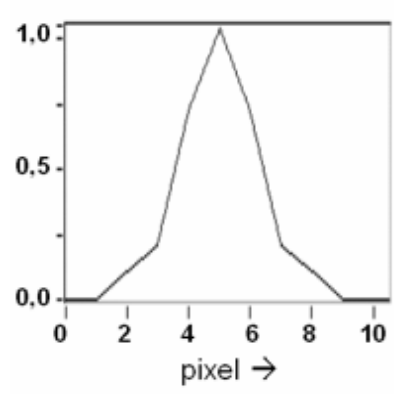

focal profile

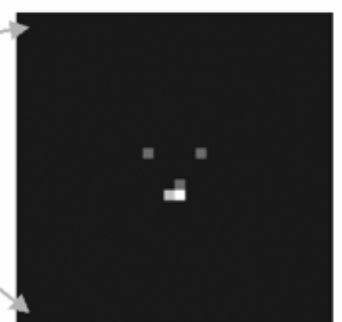

a) model section

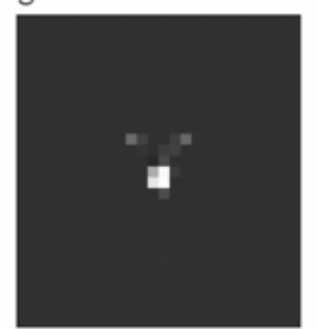

d) DIRECTT rec. 80 iterations $>8^{2}$ - fold resolution!

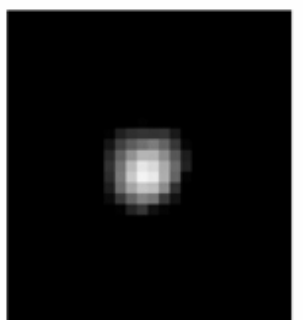

b) Fourier-filtered back projection

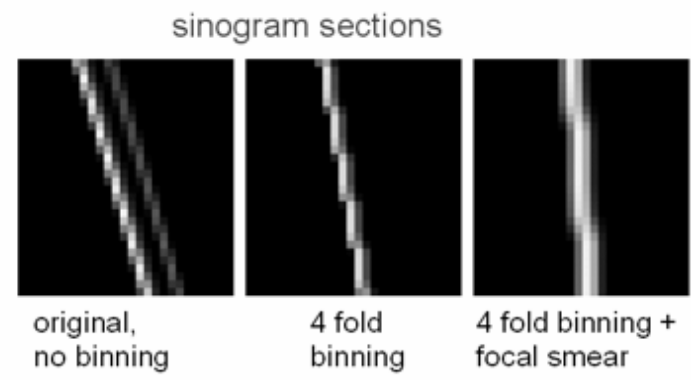

Figure 10. Sub-pixel resolution and focus desmearing 


\section{Binary reconstruction}

In Fig.11 a binary reconstruction from limited view of a triangle and $90 \mathrm{deg}$ of projections is shown. With one iteration the result looks as usual with one edge not reconstructed.On knowing the density of the object, it is possible to iteratively reconstruct the whole structure even with details, that are not seen in the projections directly.

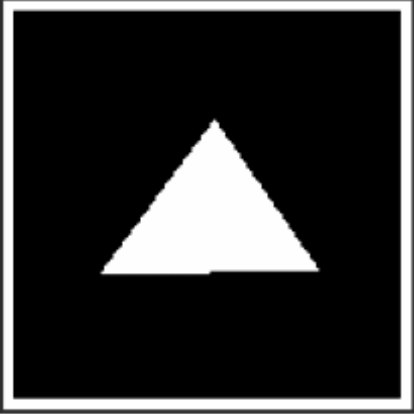

model, $256^{2}$ pixel,

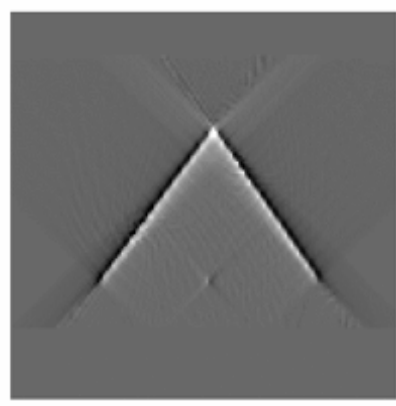

DIRECTT rec.

1 iteration, contrast

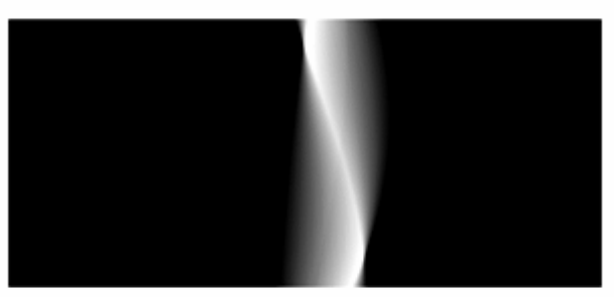

sinogram, $180 \times 0,5 \mathrm{deg}$.
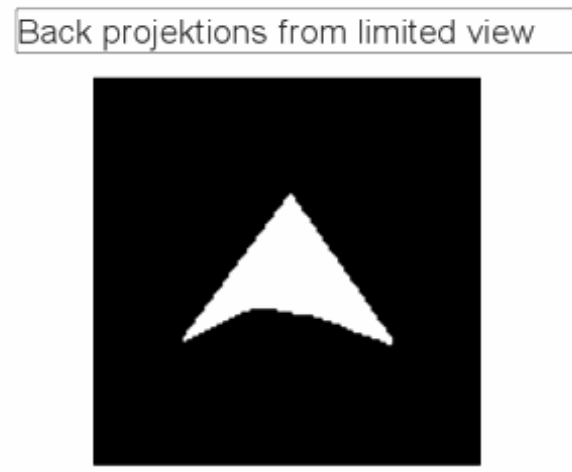

DIRECTT intermediate rec., binary density

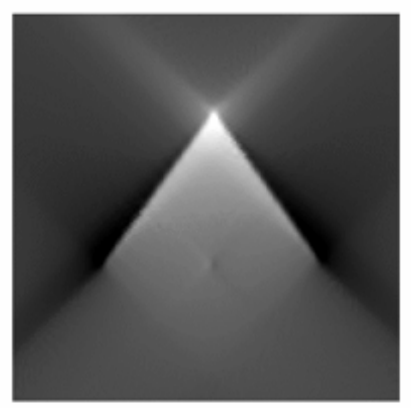

filtered back proj.

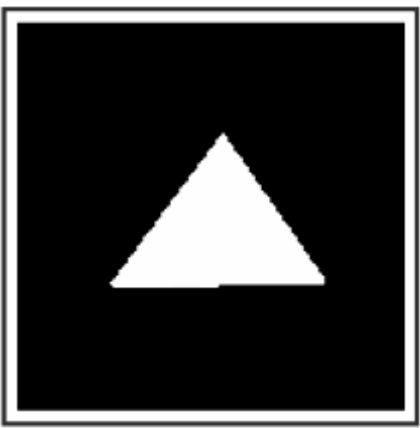

DIRECTT, 800 iterations binary density

Figure 11. Binary reconstruction from limited view data set.

\section{Measurement}

In order to evaluate the performance of the new reconstruction algorithm, the projection data of parallel beam projections are selected from a three-dimensional measurement of a metal matrix composite (MMC) sample. A $3.6 \mathrm{~mm}$ cylinder of Ti matrix containing $140 \mu \mathrm{m}$ ceramic mono-crystalline SiC fibers MMC (MTU Aero Engines) is investigated by monochromatic $39 \mathrm{keV}$ parallel Synchrotron radiation. 360 projections at $1^{\circ}$ steps are extracted from a line of a two-dimensional Detector representing a radial section of the cylinder. The intensities are registered on 1240 pixels of $6 \mu \mathrm{m}$ size by the fluorescent screen of a CCD camera. The fibres of $120 \mu \mathrm{m}$ diameter have a $10 \mu \mathrm{m}$ carbon core and a $10 \mu \mathrm{m}$ carbon coating which represent the details to be imaged.

Fig. 12 shows the conventional Fourier back projection (left) and the image of the DIRECTT procedure (right) for comparison. Although only $80 \%$ of the integral density of the sinogram has been reconstructed by 64 iterative cycles (reconstruction time of several hours; program not optimized) the contrast as well as the densities of the new 
type reconstruction are much more precise. Some noise from the low intensity measurement is reconstructed. However this hardly effects the edge contrast.

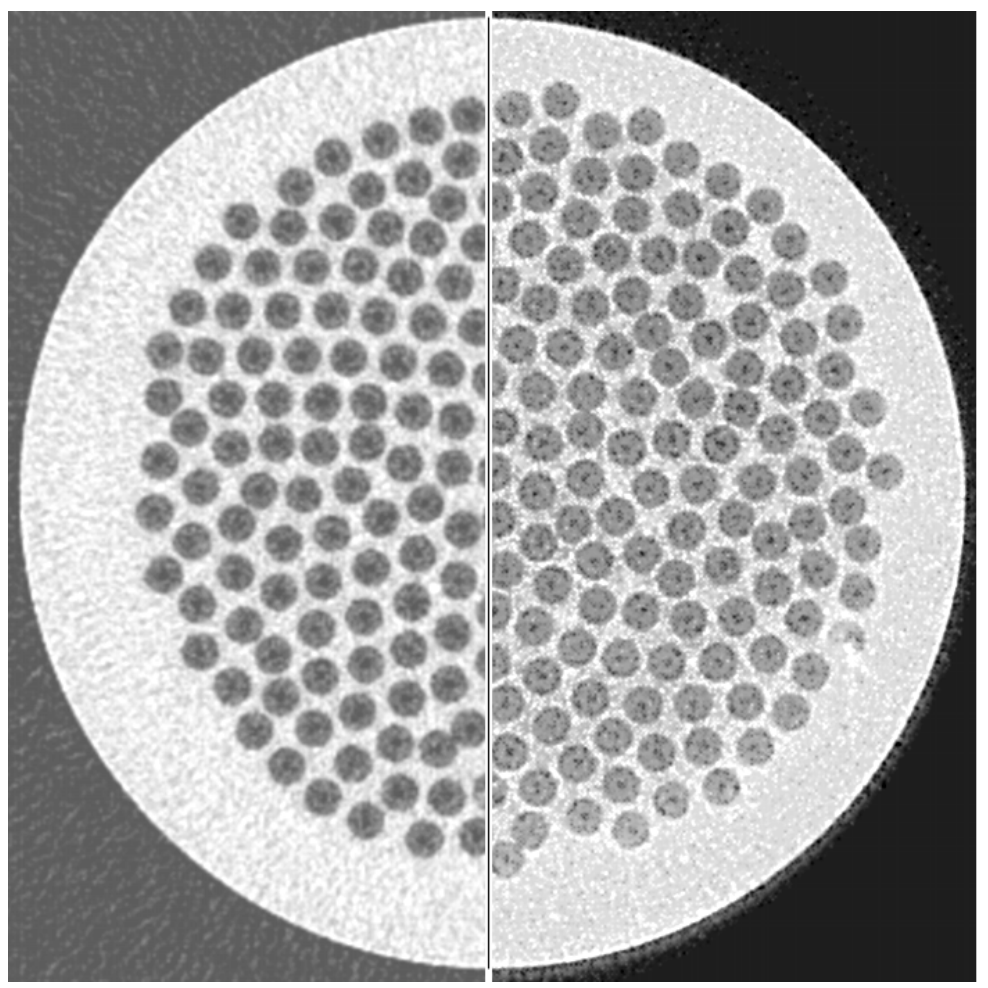

Figure 12. Comparison of reconstructions from $39 \mathrm{keV}$ parallel beam synchrotron radiation projections of $3.6 \mathrm{~mm} \mathrm{Ti} / \mathrm{SiC}$ MMC cylinder; left: conventional Fourier filtered back projection; right: DIRECTT

\section{Conclusion}

The presented reconstructions are all performed by a controlled combination of density and contrast selections and repeated artifact filtering (not discussed here). In all cases the advantages over conventional reconstructions are obvious. The resolution of the detector pixels is achieved in most cases of tested constraints. Additional advantages relate to the possible compensation for differing detector pixel response and to the selective handling of non-isotropic sample response (not investigated here), as during the iteration process all positions of the sinogram can be addressed individually. Also the principles of iteration permit to step into the process at an arbitrary level of reconstruction. Thus an additional image refinement of conventional reconstruction can be performed. Preliminary tests have also shown a considerable potential for reconstructions far beyond detector precision. For 3-D measurements the DIRECTT algorithm is not limited to a slice by slice reconstruction, as the three-dimensional projections of trajectories from very divergent projections could be reconstructed directly without major approximations.

The DIRECTT method yields high resolution images from noisy and incomplete data sets. It can be applied to all kind of projection measurements on moving and rotating objects in space. The investigations into the full performance of the algorithm are only at the beginning. 


\section{References}

1. A.C. Kak, M. Slaney, Principles of CT imaging, IEEE Press (1988) ISBN 0-87942-198-3

2. L. A. Feldkamp, L.C. Davis, J.W. Kress, Pract. cone-beam algor., J. Opt. Soc. Am., A, 1 (1984) 6, p. 612

3. H. Nyquist, Certain topics in telegraph transmission theory, Trans. AIEE, 47 (1928) pp. 617- 644

4. Patent application DE 10307331.0 (2003) and PCT/EP2004/00135, Patents pending 